\title{
MAXIMAL AND MINIMAL SOLUTIONS TO A CLASS OF ELLIPTIC QUASILINEAR PROBLEMS
}

\author{
GIOVANNI M. TROIANIELLO
}

\begin{abstract}
We prove existence of maximal and minimal solutions to bilateral problems for quasilinear elliptic operators with nondivergence principal part independent of the gradient. This result also covers the case of equations, when the obstacles can be taken as lower and upper solutions.
\end{abstract}

1. Introduction. Let $\Omega$ be a bounded open subset of $\mathbf{R}^{N}$ with a regular boundary $\partial \Omega$. For functions $u=u(x)$ satisfying inequalities of the form

$$
\left|a^{i j}\left(x, u, u_{x}\right) u_{x_{i} x_{j}}\right| \leq K\left|u_{x}\right|^{2}+g(x) \text { in } \Omega
$$

(with the usual summation convention; $u_{x} \equiv$ gradient of $u$ ), a fundamental zeroorder a priori estimate has recently been provided by Trudinger $[\mathbf{1 3}]$ and Ladyzhenskaya and Ural'ceva [9]. We state this result (which generalizes an analogous one proven by Krylov and Safonov [6, 7], cf. also [5], for solutions of linear equations) as follows.

Suppose that the functions $a^{i j}(x, u, z)$ are measurable in $\Omega$ for any fixed $(u, z) \in$ $\mathbf{R}^{1+N}$, continuous in $\mathbf{R}^{1+N}$ for almost any fixed $x \in \Omega$, and satisfy the uniform ellipticity condition

$$
\alpha|\xi|^{2} \leq a^{i j}(x, u, z) \xi_{i} \xi_{j} \leq \alpha^{-1}|\xi|^{2} \quad \forall \xi \in \mathbf{R}^{N}
$$

with a suitable positive constant $\alpha$. If a function $u$ from the Sobolev space $W_{N}^{2}(\Omega)$, hence continuous on $\bar{\Omega}$, satisfies (1) with $K \in \mathbf{R}_{+}, g \in L_{N}(\Omega)$ and vanishes on $\partial \Omega$, then $u$ verifies a Hölder condition

$$
|u(x)-u(y)| \leq L|x-y|^{2} \quad \text { for } x . y \in \bar{\Omega} .
$$

with $\gamma \in] 0,1\left[\right.$ and $L>0$ dependent only on a. $K$. $\|g\|_{L_{N}(\Omega)} .\|u\|_{L_{\infty}(\Omega)}$.

Thus the "second step" in the existence theory for a wide class of quasilinear elliptic equations of the general form

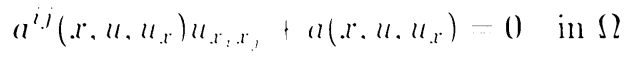

can be performed, once an a priori bound on $\|u\|_{L_{\ldots}(s 2)}$ has been obtained ("first step"). It is worth stressing that the "third step". consisting of an a priori bound on $\left\|\left|u_{x}\right|\right\|_{L_{\infty}(\Omega)}$, can be taken for solutions of (3) by following a method, originally Bernstein's, which requires additional differentiability hypotheses on the functions $a^{i j}, a$ (cf. [13, Corollary 13|). Such an implementation of the regularity assumptions is, however, no longer needed if (3) is replaced by the less general quasilinear equation

$$
a^{i . j}(x, u) u_{x_{1}, r_{,}}+a\left(x, u_{,} u_{x}\right)=0 \text { in } \Omega .
$$

Received by the editors December 6,1982 .

1980 Mathematics Subject Classification. Primary 35J65, 35R45.

Key words and phrases. Quasilinear elliptic operators, bilateral problems.

(c) 1984 American Mathematical Society $0002-9939 / 84 \$ 1.00+\$ .25$ per page 
Indeed, an estimate of type (2) for solutions of (4) almost directly leads to an $L_{\infty}$ bound on $\left|u_{x}\right|$ through a powerful technique of the semilinear theory (which concerns leading coefficients $a^{i j}=a^{i j}(x)$; cf. [2]). There is nothing too surprising about this, though we are not familiar with any explicit illustration of the matter in the existing literature. We provide one in the present paper.

What we are actually dealing with here is a slightly more general question, in that we replace (4) by the analog, for the nonvariational setting, of a bilateral variational inequality:

$$
\left\{\begin{array}{l}
\varphi \leq u \leq \psi \\
{\left[a^{i j}(x, u) u_{x_{i} x_{j}}+a\left(x, u, u_{x}\right)\right](u-\varphi) \geq 0} \\
{\left[a^{i j}(x, u) u_{x_{i} x_{j}}+a\left(x, u, u_{x}\right)\right](u-\psi) \geq 0 \text { in } \Omega}
\end{array}\right.
$$

with $\varphi=\varphi(x), \psi=\psi(x)$ given bounded functions. We prove the existence of solutions (Theorem 1), and even of maximal and minimal solutions (Theorem 2), for (5) under boundary condition

$$
u=0 \quad \text { on } \partial \Omega \text {. }
$$

It is then an easy matter to arrive at the existence of maximal and minimal solutions to (4), (6) (Theorem 3) in the case when $\varphi$ and $\psi$ can be taken to be lower and upper solutions, respectively; cf. [11] for existence results concerning semilinear divergence form operators. Notice that the bound on $\|u\|_{L_{\infty}(\Omega)}$ is now trivialized through the inequalities on the first line of (5), so that our argument does not require any use of either truncation operators or Bony's maximum principle. This allows us to handle rather general assumptions, which are weaker, even for what concerns only the first-order nonlinear term $a$, than those utilized in $[2]$ and in $[\mathbf{4}]$ for semilinear equations (see Remark 1 at the end of the paper).

2. A gradient bound. As pointed out previously, $L_{\infty}$ bounds on $\left|u_{x}\right|$ for functions $u$ satisfying

$$
\left|a^{i j}(x, u) u_{x_{i} x_{j}}\right| \leq K\left|u_{x}\right|^{2}+g(x) \text { in } \Omega
$$

follow from the Hölder bounds (2). To be precise, we let

$$
g \in L_{q}(\Omega)
$$

$q$ being fixed throughout the rest of this paper under the requirement

$$
q>N \text {. }
$$

We further assume that

$$
a_{i j} \in C^{0}(\bar{\Omega} \times \mathbf{R}) \quad \forall i, j=1, \ldots, N
$$

with

$$
\alpha|\xi|^{2} \leq a^{i j}(x, u) \xi_{i} \xi_{j} \leq \alpha^{-1}|\xi|^{2} \quad \forall \xi \in \mathbf{R}^{N}
$$

$\alpha>0$. If $u \in W_{q}^{2}(\Omega)$ satisfies (7), then (2) is valid with $\gamma$ and $L$ dependent on $u$ only via $\|u\|_{L_{\infty}(\Omega)}$. But then the moduli of continuity on $\bar{\Omega}$ of the functions $x \mapsto a^{i j}(x, u(x))$ also depend on $u$ only via $\|u\|_{L_{\infty}(\Omega)}$, and therefore the same is true for the constant $c_{1}$ of the estimate

$$
\|u\|_{W_{q}^{2}(\Omega)} \leq c_{1}\left\|a^{i j}(\cdot, u) u_{x_{i} x_{j}}\right\|_{L_{q}(\Omega)}
$$


(cf. [8]). Following the development in $[\mathbf{4}]$ of a technique introduced by Tomi, we now write $(7)$ as

$$
a^{i j}(x, u) u_{x_{i} x_{j}}=\eta(x)\left[1+\left|u_{x}\right|^{2}+g(x)\right] \text { in } \Omega,
$$

where $\|\eta\|_{L_{\infty}(\Omega)} \leq \max (K, 1)$, and solve

$$
\begin{aligned}
& v \in W_{q}^{2}(\Omega), \\
& a^{i j}(x, u) v_{x_{i} x_{j}}=\eta(x)[1+g(x)] \text { in } \Omega, \\
& v=0 \text { on } \partial \Omega .
\end{aligned}
$$

Thanks to the continuous imbedding of $W_{q}^{2}(\Omega)$ in $C^{1+\delta}(\bar{\Omega})$ for a suitable $\left.\delta \in\right] 0,1[$, the estimate

$$
\|v\|_{W_{q}^{2}(\Omega)} \leq c_{1}\left\|a^{i j}(\cdot, u) v_{x_{i} x_{j}}\right\|_{L_{q}(\Omega)}
$$

yields a bound of the same type as above on the $L_{\infty}$ norm of $\left|v_{x}\right|$, hence also on the $L_{\infty}$ norm of the function

$$
\theta \equiv \eta\left|u_{x}\right|^{2} /\left(1+\left|u_{x}-v_{x}\right|^{2}\right)
$$

Let $z \equiv u-v$ : since $z \in W_{q}^{2}(\Omega)$ satisfies

$$
\begin{aligned}
a^{i j}(x, u) z_{x_{i} x_{j}} & =\theta(x)\left(1+\left|z_{x}\right|^{2}\right) \quad \text { in } \Omega, \\
z & =0 \text { on } \partial \Omega,
\end{aligned}
$$

the same technique as in $[\mathbf{2}]$ yields a bound on $\left\|\left|z_{x}\right|\right\|_{L_{\infty}(\Omega)}$, hence on $\left\|\left|u_{x}\right|\right\|_{L_{\infty}(\Omega)}$, which depends on $u$ only via the moduli of continuity of the functions $a^{i j}$. Thus we have the following

LEMMA. Let the inequality (7), under assumptions (8)-(11), hold true for a function $u \in W_{q}^{2}(\Omega)$ vanishing on $\partial \Omega$. Then there exists a constant $c_{2}$, dependent only on $\alpha, K,\|g\|_{L_{q}(\Omega)}$ and $\|u\|_{L_{\infty}(\Omega)}$ as well as on the moduli of continuity of the functions $a^{i j}$ on $\bar{\Omega} \times[-C, C]\left(C \geq\|u\|_{L_{\infty}(\Omega)}\right)$, such that $\left\|\left|u_{x}\right|\right\|_{L_{\infty}(\Omega)} \leq c_{2}$.

3. Existence of solutions. We now pass to the existence result for (5), (6). The function $a: \Omega \times \mathbf{R} \times \mathbf{R}^{N} \rightarrow \mathbf{R}$ is assumed to satisfy:

$$
\begin{gathered}
x \mapsto a(x, u, z) \text { is measurable for any }(u, z), \\
(u, z) \mapsto a(x, u, z) \text { is continuous for a.a. } x, \\
|a(x, u, z)| \leq K|z|^{2}+g(x) \text { in } \Omega \times[-C, C] \times \mathbf{R}^{N}, \\
C \in \mathbf{R}_{+}(K \text { and } g \text { possibly varying with } C) .
\end{gathered}
$$

As for the obstacles $\varphi$ and $\psi$, they are of the form

$$
\begin{aligned}
& \varphi \equiv \bigvee_{h=1}^{\mu} \varphi^{h} \quad \text { with } \varphi^{h} \in W_{q}^{2}(\Omega), \varphi^{h} \leq 0 \text { on } \partial \Omega, \\
& \psi \equiv \bigwedge_{k=1}^{\nu} \psi^{k} \quad \text { with } \psi^{k} \in W_{q}^{2}(\Omega), \psi^{k} \geq 0 \text { on } \partial \Omega
\end{aligned}
$$

and satisfy

$$
\varphi \leq \psi \quad \text { on } \bar{\Omega} .
$$


THEOREM 1. Under assumptions (8)-(11) and (13)-(18), problem (5), (6) admit a solution $u \in W_{q}^{2}(\Omega)$.

We simply sketch the proof, since it follows the same pattern as in the semilinear case (for parabolic operators, cf. $[\mathbf{1 2}]$ ). For any $\tau \in[0,1]$ and any $v \in C^{1}(\bar{\Omega})$ we consider the problem: find $\omega \in W_{q}^{2}(\Omega)$ such that

$$
\left\{\begin{array}{l}
\tau \varphi \leq \omega \leq \tau \psi, \\
{\left[a^{i j}(x, v) \omega_{x_{i} x_{j}}+a\left(x, v, v_{x}\right)\right](\omega-\tau \varphi) \geq 0} \\
{\left[a^{i j}(x, v) \omega_{x_{i} x_{j}}+a\left(x, v, v_{x}\right)\right](\omega-\tau \psi) \geq 0 \quad \text { in } \Omega} \\
\omega=0 \text { on } \partial \Omega .
\end{array}\right.
$$

It is easy to see that the (singlevalued) mapping $T:(\tau, v) \mapsto \omega$ so defined is continuous and compact from $[0,1] \times C^{1}(\bar{\Omega})$ into $C^{1}(\bar{\Omega})$. Moreover, $T(0, v)=0 \forall v \in C^{1}(\bar{\Omega})$. The Leray-Schauder fixed point theorem can be applied to the mapping $T(1, \cdot)$, and the existence of a solution to our problem can thus be proven, if an a priori bound on $\left\|\left|v_{x}\right|\right\|_{L_{\infty}(\Omega)}$ is provided for all possible fixed points $v=T(\tau, v), \tau \in[0,1]$. But any such function $v$ satisfies the inequalities of the Lewy-Stampacchia type

$$
\begin{gathered}
{\left[\tau \bigwedge_{h=1}^{\mu} a^{i j}(x, v) \varphi_{x_{i} x_{j}}^{h}\right] \wedge\left[-a\left(x, v, v_{x}\right)\right] \leq a^{i j}(x, v) v_{x_{i} x_{j}}} \\
\leq\left[\tau \bigvee_{k=1}^{\nu} a^{i j}(x, v) \psi_{x_{i} x_{j}}^{k}\right] \vee\left[-a\left(x, v, v_{x}\right)\right] \text { in } \Omega
\end{gathered}
$$

which imply

$$
\left|a^{i j}(x, v) v_{x_{i} x_{j}}\right| \leq \sum_{h=1}^{\mu}\left|a^{i j}(x, v) \varphi_{x_{i} x_{j}}^{h}\right|+\sum_{k=1}^{\nu}\left|a^{i j}(x, v) \psi_{x_{i} x_{j}}^{k}\right|+2\left|a\left(x, v, v_{x}\right)\right| .
$$

Since the functions $a^{i j}(x, v)$ can be bounded in $L_{\infty}(\Omega)$ independently of $v$ (thanks to the automatic bound on the latter ensured by the constraints), an inequality

$$
\left|a^{i j}(x, v) v_{x_{i} x_{j}}\right| \leq \hat{K}\left|v_{x}\right|^{2}+\hat{g}(x) \quad \text { in } \Omega
$$

with $\hat{g} \in L_{q}(\Omega)$, follows from (15) and (19). Then the lemma above yields the sought-for $L_{\infty}$ bound on $\left|v_{x}\right|$.

4. Maximal and minimal solutions. The possibility of solving (5), (6) for obstacles having the slightly complicated expressions (16) and (17) is utilized in the proof of our next result.

THEOREM 2. Under the same assumptions of Theorem 1, problem (5), (6) admit a maximal and a minimal solution.

To prove this theorem we adapt Akô's method (cf. $[\mathbf{1}, \mathbf{3}]$ ). We define

$$
\begin{aligned}
& u_{\max }(x) \equiv \sup \{u(x) \mid u \text { solves }(5),(6)\}, \\
& u_{\min }(x) \equiv \inf \{u(x) \mid u \text { solves }(5),(6)\},
\end{aligned}
$$

and proceed to prove that both $u_{\max }$ and $u_{\min }$ are solutions. 
Denoting by $\left\{x^{m}\right\}_{m \in \mathbf{N}}$ a countable dense subset of $\bar{\Omega}$, we construct for each $m$ a sequence $\left\{u^{m, n}\right\}_{n \in \mathbf{N}}$ of solutions to (5), (6) such that

$$
u_{\max }\left(x^{m}\right)=\lim _{n \rightarrow \infty} u^{m, n}\left(x^{m}\right) .
$$

Then we consider (5) with $\varphi$ replaced by $u^{1,1}$ and correspondingly find a solution $U^{1}$ satisfying (6). The subset $\left\{U^{1}>\varphi\right\}$ of $\Omega$ where $U^{1}$ is strictly larger than $\varphi$ can be decomposed into $\left\{U^{1}>u^{1,1}\right\} \cup\left\{U^{1}=u^{1,1}>\varphi\right\}$. In $\left\{U^{1}>u^{1,1}\right\}$ the inequality

$$
\left[a^{i j}\left(x, U^{1}\right) U_{x_{i} x_{j}}^{1}+a\left(x, U^{1}, U_{x}^{1}\right)\right]\left(U^{1}-u^{1,1}\right) \geq 0
$$

yields

and therefore also

$$
a^{i j}\left(x, U^{1}\right) U_{x_{i} x_{j}}^{1}+a\left(x, U^{1}, U_{x}^{1}\right) \geq 0,
$$

$$
\left[a^{i j}\left(x, U^{1}\right) U_{x_{i} x_{j}}^{1}+a\left(x, U^{1}, U_{x}^{1}\right)\right]\left(U^{1}-\varphi\right) \geq 0 .
$$

In $\left\{U^{1}=u^{1,1}>\varphi\right\}, u^{1,1}$ verifies

$$
a^{i j}\left(x, u^{1,1}\right) u_{x_{i} x_{j}}^{1,1}+a\left(x, u^{1,1}, u_{x}^{1,1}\right) \geq 0,
$$

whereas, by a well-known result due to Stampacchia,

$$
U_{x_{i}}^{1}=u_{x_{i}}^{1,1}, \quad U_{x_{i} x_{j}}^{1}=u_{x_{i} x_{j}}^{1,1} \text { for } i, j=1, \ldots, N,
$$

so that (21) is again satisfied. This shows that (21) is valid throughout $\Omega$, hence that $U^{1}$ is still a solution of $(6)$.

We can inductively define a sequence $\left\{U^{p}\right\}_{p \in \mathbf{N}} \subset W_{q}^{2}(\Omega)$, where $U^{p}$ solves (5) with $\varphi$ replaced by $\bigvee_{i=1}^{p} u^{i, p} \vee U^{p-1}$ (admissible because of the form (16)) and satisfies (6). Since

$$
\begin{aligned}
\left\{U^{p}>\varphi\right\}= & \left\{U^{p}>\bigvee_{i=1}^{p} u^{i, p} \vee U^{p-1}\right\} \cup\left\{U^{p}=u^{1, p}>\varphi\right\} \\
& \cup \cdots \cup\left\{U^{p}=u^{p, p}>\varphi\right\} \cup\left\{U^{p}=U^{p-1}>\varphi\right\},
\end{aligned}
$$

an analysis as above shows that, if $U^{p-1}$ solves (5), then $U^{p}$ satisfies

$$
\left[a^{i j}\left(x, U^{p}\right) U_{x_{i} x_{j}}^{p}+a\left(x, U^{p}, U_{x}^{p}\right)\right]\left(U^{p}-\varphi\right) \geq 0
$$

and is therefore a solution of (5).

By proceeding as in the proof of Theorem 1 we can now see that the $U^{p}$ 's are uniformly bounded in $C^{1}(\bar{\Omega})$ and therefore also in $W_{q}^{2}(\Omega)$ (cf. the discussion of the bounds (12), (20)). By monotonicity, the whole sequence converges weakly in $W_{q}^{2}(\Omega)$, hence strongly in $C^{1}(\bar{\Omega})$, toward a function $U$; a passage to the limit in (5) written for $u \equiv U^{p}$ shows that $U$ is a solution of (5), (6). Since

$$
\lim _{p \rightarrow \infty} U^{p}\left(x^{m}\right) \equiv u_{\max }\left(x^{m}\right) \quad \forall m \in \mathbf{N},
$$

$U$ satisfies

$$
U\left(x^{m}\right) \geq u\left(x^{m}\right) \quad \forall m \in \mathbf{N},
$$

and therefore by density

$$
U(x) \geq u(x) \quad \forall x \in \bar{\Omega},
$$

whenever $u$ is a solution to our problem. Hence throughout $\bar{\Omega}, U$ equals $u_{\max }$, and the latter is a solution. The proof concerning $u_{\min }$ is analogous. 
5. The equation. In order to pass to the study of (4) we recall that a function $\varphi$ of the form (16) (a function $\psi$ of the form (17)) is a lower (an upper) solution of (4) if

$$
\begin{aligned}
a^{i j}\left(x, \varphi^{h}\right) \varphi_{x_{i} x_{j}}^{h}+a\left(x, \varphi^{h}, \varphi_{x}^{h}\right) \geq 0 \quad \forall h=1, \ldots, \mu \\
\left(a^{i j}\left(x, \psi^{k}\right) \psi_{x_{i} x_{j}}^{k}+a\left(x, \psi^{k}, \psi_{x}^{k}\right) \leq 0 \forall k=1, \ldots, \nu\right) .
\end{aligned}
$$

In particular, almost everywhere in any nonvoid set where a function $\varphi^{h}$ equals a function $\psi^{k}$, both functions satisfy the equation. We have

THEOREM 3. In addition to the hypotheses of Theorem 1 , let $\varphi$ and $\psi$ be a lower and an upper solution, respectively. Then the set of all solutions to (4), (6) which lie between $\varphi$ and $\psi$ coincides with the set of all solutions to (5), (6); therefore, it is not empty and admits a maximal and a minimal solution.

In order to prove the theorem it suffices to notice that any solution of (5) automatically solves (4) in the subset of $\Omega$ where $\varphi<u<\psi$, whereas, for instance, in the subset of $\Omega$ in which $u=\varphi^{h}<\psi$ the following inequalities hold:

$$
\begin{aligned}
0 & \geq a^{i j}(x, u) u_{x_{i} x_{j}}+a\left(x, u, u_{x}\right) \quad(\text { third line of }(5)) \\
& =a^{i j}\left(x, \varphi^{h}\right) \varphi_{x_{i} x_{j}}^{h}+a\left(x, \varphi^{h}, \varphi_{x}^{h}\right) \geq 0 .
\end{aligned}
$$

REMARK 1. In the semilinear case $a^{i j}=a^{i j}(x)$, the method of lower and upper solutions has, for instance, been utilized by Kazdan and Kramer [4] and Amann and Crandall [2]. In the former paper, existence of solutions is proven under the additional assumption that $a(x, u, z)=\alpha(x, u)+\beta(x, u, z)$ with

$$
\beta \in C^{0}\left(\bar{\Omega} \times \mathbf{R} \times \mathbf{R}^{N}\right)
$$

in the latter, existence of maximal and minimal solutions is proven under the additional assumption that $a, a_{u}$ and $a_{z}$ are continuous in $\bar{\Omega} \times \mathbf{R} \times \mathbf{R}^{N}$.

REMARK 2. The results above obviously cover the case of a quasilinear divergence form operator $\left(a^{i j}(x, u) u_{x_{i}}\right)_{x_{j}}+a\left(x, u, u_{x}\right)$ when

$$
\left|a_{x}^{i j}\right| \in L_{2 q}(\Omega \times]-C, C[), \quad a_{u}^{i j} \in L_{\infty}(\Omega \times]-C, C[)
$$

with $C>0$ suitably large. Besides, our techniques and results can be extended with no difficulty to parabolic operators, thanks to the parabolic version of the zero-order estimate (cf. $[\mathbf{1 0}])$.

\section{REFERENCES}

1. K. Akô, On the Dirichlet problem for quasi linear elliptic differential equations of second order, J. Math. Soc. Japan 13 (1961), 45-52.

2. H. Amann and M. G. Crandall, On some existence theorems for semilinear elliptic equations, Indiana Univ. Math. J. 27 (1978), 779-790.

3. J. W. Bebernes and K. Schmitt, On the existence of maximal and minimal solutions for parabolic partial differential equations, Proc. Amer. Math. Soc. 73 (1979), 211-218.

4. J. L. Kazdan and R. J. Kramer, Invariant criteria for existence of solutions to second-order quasilinear elliptic equations, Comm. Pure Appl. Math. 31 (1978), 619-645.

5. N. V. Krylov, Sequences of convex functions and estimates of maximum of solutions of parabolic equations, Siberian Math. J. 17 (1976), 290-303. 
6. N. V. Krylov and M. V. Safonov, An estimate of the probability that a diffusion process hits a set of positive measure, Soviet Math. Dokl. 20 (1979), 253-255.

7. _ Certain properties of solutions of parabolic equations with measurable coefficients, Izv. Akad. Nauk SSSR Ser. Mat. 40 (1980), 161-175.

8. O. A. Ladyzhenskaya and N. N. Ural'ceva, Linear and quasilinear elliptic equations, Academic Press, New York, 1968.

9. ___ Estimate of Hölder norm for solutions of second order quasilinear elliptic equations of general form, Uspehi Mat. Nauk 35 (1980), 144-145.

10. __ Estimates of Hölder constants for bounded solutions of second order quasilinear parabolic equations of nondivergence form, LOMI preprint E-11-81, Leningrad, 1981.

11. J. P. Puel, Existence, comportement à l'infini et stabilité dans certains problèmes quasilinéaires ellip tiques et paraboliques d'ordre 2, Ann. Sci. École Norm. Sup. (4) 3 (1976), 89-119.

12. G. M. Troianiello, Bilateral constraints and invariant sets for semilinear parabolic systems, Indiana Univ. Math. J. 32 (1983), 563-577.

13. N. S. Trudinger, Local estimates for subsolutions and supersolutions of general second order elliptic quasilinear equations, Invent. Math. 61 (1980), 67-79.

istituto Matematico "G. Castelnuovo”, Universitá di Roma i, Rome. Italy 